\title{
The Experts Speak on Biobank Education
}

\author{
Edited by Sheila O'Donoghue ${ }^{1,2}$ and Lise Matzke ${ }^{2}$ \\ Authors: Jennifer A. Byrne, ${ }^{3,4}$ Clive Green, ${ }^{5}$ Kristina Hill, ${ }^{6}$ Zisis Kozlakidis, \\ Annemieke De Wilde, ${ }^{8}$ Piper Mullins, ${ }^{9}$ and Kerry R. Wiles ${ }^{10}$
}

\section{Editors' Introduction}

$\mathrm{D}$ ESIGNING FUTURE BIOBANK EDUCATION is a challenge as there are many different types/models of biobanks, multiple stakeholders (researchers, funders, and participants), and a range of skill sets required to conduct the activity of biobanking. Learners need to continuously update their knowledge to keep up with progress in science, which results in evolving and increasingly complex biospecimen and data needs of researchers. In addition, learners need to be aware of the changes that occur with regular updates to biobanking standards, ethical guidelines, and best practices.

We asked experts from industry and academic human biobanks from Australia, Europe, and North America to share their thoughts on the following question:

If you are advising on the development of a new course on biobanking, which three topics in best practices would you most emphasize and why?

- repository planning

- facilities

- storage

- quality management

- method validation

- safety

- training

- cost management

- information management

- packing and shipping

- specimen collection and handling

- ethical legal

- specimen access.

These topics reflect the distinct and important areas of knowledge relevant to biobanking as reflected in the chapter headings of the ISBER Best Practices 2018. The experts' perspectives on which topics should be priorities varied as expected. The majority identified quality management as an important focus for future education. The second most com- monly identified priority topics for future education needs were related to ethical/legal issues, specimen access, and repository planning. Several other topics were also highlighted by at least one expert, but three topics (facilities, cost management, and packing and shipping) were not emphasized.

It should be noted that, by the nature of the question posed, the experts' responses should not be interpreted as indicating what topics are the most important in biobanking. Instead these responses should be considered as a guide for added focus on specific topics by those interested in the development of new education courses or improvements to existing courses.

Address correspondence to: Sheila O'Donoghue, RN, BPHE

Biobank Leader The Tumour Tissue Repository BC Cancer 2410 Lee Avenue Victoria, BC, V8R6V5

Canada

Project Manager Office of Biobank Education and Research (OBER) Department of Pathology and Laboratory Medicine University of British Columbia Vancouver V6T $2 B 5$ Canada

E-mail: sodonoghue@pathology.ubc.ca

Lise Matzke, MSc, MBA

Operations and Business Lead

Office of Biobank Education and Research (OBER)

Department of Pathology and Laboratory Medicine University of British Columbia

Vancouver V6T $2 B 5$

Canada

E-mail: 1matzke@pathology.ubc.ca

\footnotetext{
${ }^{1}$ Tumour Tissue Repository, Deeley Research Center, BC Cancer, Victoria, Canada.

${ }^{2}$ Office of Biobank Education and Research (OBER), Department of Pathology and Laboratory Medicine, University of British Columbia, Vancouver, Canada.

${ }^{3}$ NSW Health Statewide Biobank, Camperdown, NSW, Australia.

${ }_{5}^{4}$ Faculty of Medicine and Health, The University of Sydney, Australia.

${ }^{5}$ Discovery Sciences, Biopharmaceuticals R\&D, AstraZeneca, Cambridge, United Kingdom.

${ }^{6}$ Independent Consultant, Florida.

${ }^{7}$ Laboratory Services and Biobank Group, International Agency for Research on Cancer (IARC), Lyon, France.

${ }^{8}$ Biobank Antwerpen, Edegem, Belgium.

${ }^{9}$ Pan-Smithsonian Cryo-Initiative, Smithsonian Institution, Washington, District of Columbia.

${ }^{10}$ Vanderbilt University Medical Center, Nashville, Tennessee.
} 


\section{Expert Response: Jennifer A. Byrne}

B IOBANKS EXIST TO SUPPORT RESEARCH, and provide a vital link between patients and researchers. Regular training allows biobank staff to maintain and ultimately improve biobanking standards, so that the results of supported research are more likely to be robust and reproducible. I found it difficult to choose between the listed training priorities, as all nominated areas are important. However, if obliged to choose, I would prioritize safety, ethical legal, and quality management.

Safety must be chosen as it is always paramount. Biobank staff members need to learn how to work safely, and how to play their part in creating and maintaining a safe work environment. Safety training requires regular updates as risks, infrastructure, and regulations all change over time. Similarly, all biobank staff members need to understand the relevant ethical and legal requirements that underpin their work, which are also subject to change. It is not acceptable to delegate this responsibility to biobank leaders, who may not always be available to provide advice. Finally, knowledge of the principles of quality should underpin every aspect of biobanking. When biobank staff members commit to upholding standards through quality management training, these principles can be extended to every aspect of biobanking.

Regardless of the areas chosen, I would add that wherever possible, biobank staff training should be free of charge, and accessible to all staff members, regardless of whether they are working full- or part-time. Online training is convenient, but face-to-face training allows for questions and discussions, and can also promote networking between biobanks and researchers. For example, general biosafety training sessions may usefully serve biobank staff, and also provide an opportunity to meet local laboratory researchers in person. Finally, I have also heard that consuming cake during training sessions promotes information retention, but I understand that this requires further participatory research.

Address correspondence to: Jennifer A. Byrne, BSc(Hons), PhD Director of Biobanking NSW Health Statewide Biobank Level 1, 67-73 Missenden Road Camperdown, NSW 2050 Australia

Professor of Molecular Oncology Faculty of Medicine and Health The University of Sydney Australia

E-mail: jennifer.byrne@health.nsw.gov.au

\section{Expert Response: Clive Green}

$\mathrm{T}$ HE SUCCESS OF A BIOBANK is highly dependent on its ability to demonstrate value to key stakeholder groupsscientific users, patients, and funders. This requires long range planning based on understanding the requirements of scientists, maximizing the accessible patient cohort, and defining a sound financial operating model.
It is imperative that enough time is dedicated to understanding the user community and their specific requirements. Increasingly, a high-quality collection of primary disease tissue biospecimens is considered an entry-level requirement. Scientists attach value to collections that offer additional characteristics to drive exploratory research-for example, matched blood samples, control samples, samples from longitudinal studies, and health record data.

A biobank should understand the profile of scientific users across industry and academia, and actively work to serve both groups. Focus should be placed on understanding the research areas of drug companies and targeting those whose disease area science aligns with the accessible patient cohorts. Effort should also be invested to understand the financial value companies place on the specific biospecimen types that can be supplied.

Finally, a cost recovery model is essential, since it ensures a biobank can establish a degree of financial security. This model should include costs aligned with long-term investments in the biobank and a plan to achieve financial independence from funding bodies. A biobank should also understand how it wishes to align these costs to different scientific user groups, for example, zero-based costing for academic users compared with fully loaded costs for industry users.

Taken together, a long-term plan that applies detailed consideration to these key areas can enable a biobank to play a key role in bringing new and life-changing medicines to patients.

Address correspondence to: Clive Green, PhD

Executive Director, Sample Management Discovery Sciences

Biopharmaceuticals $R \& D$

AstraZeneca

Cambridge CB4 OWG

United Kingdom

E-mail: clive.green@astrazeneca.com

\section{Expert Response: Kristina Hill}

口 Ach of the 13 ISBER Best Practice modules is Eessential to the successful functioning of any repository, in addition to the biobankers or repository technicians that work within. Technicians are at the heart of repositories and must possess basic skills for the day-to-day activities that may include processing, shipping, and quality control. That being said, there are additional aspects of repositories that technicians, in addition to the repository director and manager, should be familiar with. Repository planning is crucial for defining the repository's purpose and the creation of a governance plan. In most cases, this is not considered until after a repository has been created or already exists, but is vital for any technicians wishing to advance in their career and understand the basics of repository inception and function. Legal and ethical issues can be very complex and come across more effectively when communicated or explained by experts, especially for the nuances encountered with regional or local laws/ regulations. A good understanding of these concepts can 
better explain/describe the repository as a complete entity. Specimen access is another complex area where a technician would benefit from additional education/training. Transfer agreements, appropriate specimen uses, and culling encompass how the entire repository functions even after specimens have been processed and shipped.

Repository technicians can benefit from this knowledge to help with career advancement and gain a better understanding of all facets of the repository. On-the-job training may not include this information and it is more valuable for these larger overarching topics to be explained, or taught by experts in a more relatable way, as opposed to strictly learning the information from a slide set or document. The entire repository thrives when staff members possess the complete set of skills and knowledge available to them.

Address correspondence to: Kristina Hill, MPH, BSc MT Independent Consultant Florida

E-mail: hillke3@gmail.com

\section{Expert Response: Zisis Kozlakidis}

T I WAS ADVISING on the development of a new course on biobanking for technicians, the following three topics in best practices would be emphasized: quality management, information management, and specimen collection and handling.

Currently there is no specific training course for technicians in the field of biobanking. Rather technicians tend to happen upon biobanking, or to follow practices for a number of years only to realize at some later point that the continuum of protocols, from collecting, processing, and storing to distributing samples and data, is called "biobanking." In my view, such a course is long overdue for the field.

Having said that, technicians already should have some training in relation to specimen collection and handling, not necessarily to the standard required for biobanking. As such I would consider this as the essential foundation, so that the specimen collection and handling processes are seen from the perspective of biobanking.

The quality management follows quite naturally from that, as it provides the evidence base that links the practice to the impact of biobanking. It is crucial for the sustainability of any biobank that high-quality samples are being collected and provided, not only in the narrow sense of financial sustainability but also in the wider sense of scientific and social sustainability.

Because of the exponential increase in the volume and veracity at which data are produced and linked to physical samples, I believe the information management aspect will become a core indispensable aspect of biobanking, and as such needs to be included in any training course. Technicians are not "data-immune" or "data-agnostic" any longer, quite the opposite they need to become data savvy in the field of biobanking. Large biobanks are already facing big data challenges; technicians are part of the picture and need to be appropriately trained and prepared for these challenges.

\author{
Address correspondence to: \\ Zisis Kozlakidis, PhD \\ Group Head \\ Laboratory Services and Biobank Group \\ International Agency for Research on Cancer (IARC) \\ 150 Cours Albert Thomas \\ 69372 Lyon CEDEX 08 \\ France \\ E-mail: kozlakidisz@iarc.fr
}

\section{Expert Response: Mieke De Wilde}

A $S$ The DAIly JOB OF TECHNiCIANS is very practical, it

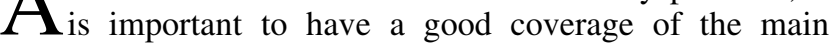
biobank processes. These topics are, for example, addressed in the ISBER best practices sections: "Specimen Collection and Handling," "Specimen Access," and "Packaging and Shipping." The management of associated data is addressed in the section "Repository Information Management Systems." Although these sections are very important, I would most emphasize the sections "Ethical and Legal Issues," "Quality Management," and "Method Validation and Quality Control."'

Biobankers need to be aware that what they are doing is made possible through generous gifts of human donors. Focus on the "Ethical and Legal Issues" section is an absolute requirement to ensure that this human material is handled according to the highest ethical and legal standards, including regional, national, and international legislation and requirements.

Reliable and reproducible research results are very important for scientific advancement and health care research. Biobanks need to have quality assurance and quality control measures in place, using evidence-based standard methods to provide samples and data that are fit for the intended research purpose. Continuous improvement of the biobank preanalytic processes will address the concerns and needs of stakeholders such as donors, researchers, and funders. Special attention should, therefore, be given to the sections "Quality Management" and "Method Validation and Quality Control."

Knowledge and education in biobank quality are moving forward. In 2018, ISO20387: "General requirements for biobanking" was published. Additional international standards are being developed. Among them are document 22758: "Implementation guide for ISO20387,", and document 21899, which addresses method validation. The American Society for Clinical Pathology-Board of Certification (ASCP-BOC) and ISBER initiative on the development of a Qualification in BioRepository Science (QBRS) certification for biobank technicians, enables biobankers to gain recognition for their competences.

Address correspondence to:
Annemieke De Wilde, MS
Quality Manager
Biobank Antwerpen
Clinical Research Center
UZA/UAntwerpen
Wilrijkstraat 10
Edegem 2650
Belgium E-mail: annemieke.dewilde@uza.be 


\section{Expert Response: Piper Mullins}

S $\mathrm{S}$ the program Manager for the Pan-Smithsonian A Cryo-Initiative, I advise laboratories on managing frozen biospecimens. Our frozen collections house a variety of animal, plant, and environmental biospecimens. Although the sample content may vary between animal and human biospecimens, the operations remain largely the samefreezers store biospecimens in appropriate containers, and databases store the biospecimen inventory and metadata. We, therefore, educate our technicians to understand biobank operational activities. Accordingly, I recommend developing biobank courses that emphasize best practices related to regular technician tasks: specimen handling, information management, and storage.

Foremost, I recommend a course curriculum that highlights specimen collection and handling. Although it is a broad category, it is an integral part of daily biobank tasks. Best practices emphasize the elements responsible for maintaining specimen integrity, effects of freeze-thaw cycles, and appropriate storage containers. Technicians familiar with these elements are prepared to draft appropriate standard operating procedures (SOPs) that fit within the workflow of their biobank. A biobank course presenting these broad specimen handling elements may complement as-needed training for specific biobank protocols.

A biobank course should also feature information management best practices, as biobanks need technicians who can validate data to ensure effective tracking systems. In particular, I recommend emphasizing a general knowledge of specimen descriptors and show how to determine those necessary for specific projects or biobanks. I also advise to include information management reference standards specific to the intended audience's industry.

Another important element to biobank education is storage systems knowledge. Technicians are usually the first responders to freezer emergencies. They should be trained in monitoring machines and facilities, for mechanical, liquid nitrogen, and walk-in storage systems. As staff who work daily with storage systems, technicians familiar with storage best practices are able to mitigate problems and recommend solutions specific to their biobank facility.

A biobank course that emphasizes these three elements of daily biobank operations ensures trained technicians who implement best practices to process, database, and store quality biospecimens, regardless of sample type.

Address correspondence to: Piper Mullins, MIS

Pan-Smithsonian Cryo-Initiative Smithsonian Institution

P.O. Box 37012, MRC 5502

Washington, DC 20013-7012

E-mail: mullinsp@si.edu

\section{Expert Response: Kerry R. Wiles}

S AN ACADEMIC BIOREPOSITORY DIRECTOR, we train entry-level staff members with the intention of transitioning them to broader roles as their career develops and they begin to focus on their strengths and interests. Three areas of the biorepository that are the most difficult for new technicians to grasp are:

1. The quality management system

2. Task-specific training, documentation, and auditing (SOPs)

3. Access to tissue and the ethical, legal, and social issues surrounding the release of tissue to investigators.

Courses designed to educate new technicians on how their role "fits" with the quality management system (QMS) are crucial to the biorepository success and to their own success and career advancement. The QMS is the backbone of any industry, including the biorepository, and provides clear objectives, priorities, and expectations for employees.

The biorepository is primarily designed to add value to the customer or organization by determining the needs of the customer and transforming those needs into internal requirements, such as SOPs, to produce a product or service that meets or exceeds the customer's expectation, while adhering to institutional, state, and federal regulations (ethical, legal, and social issues).

The QMS coordinates and directs these activities through the use of SOPs, training manuals and documents processes, and procedures for achieving quality, while adhering to ethical principles surrounding the release of specimens to the researcher.

When processes are standardized and documented, it takes less time and effort to train new employees and existing employees, transitioning to new roles. Technicians entering the biorepository field may not have experience with ethical principles surrounding the use of human tissue and the requirements for obtaining consent for specific research applications. Therefore, an in-depth course designed to address data sharing policies, data use agreements, material transfer agreements, confidentiality agreements, and other documentation required for the transfer of specimens and data to researchers is highly recommended.

Most biorepositories have an access-to-tissue policy; however, courses designed to address disputes that may occur over allocation decisions may be of secondary interest.

Address correspondence to:

Kerry R. Wiles, BS

Program Director

CHTN Western Division Coordinator Vanderbilt University Medical Center 1211 Medical Center Drive Nashville, TN 37232-5310

E-mail: kerry.wiles@vumc.org 